\title{
A Small-Molecule Inhibitor to the Cytokine Interleukin-4
}

Sean P. Quinnell ${ }^{1}$, Becky S. Leifer ${ }^{2,3}$, Stephen T. Nestor ${ }^{1}$, Kelly Tan ${ }^{1}$, Daniel F. Sheehy ${ }^{1}$, Luke $\mathrm{Ceo}^{1}$, Shelby K. Doyle ${ }^{2,3}$, Angela N. Koehler ${ }^{2,3,4}$, Arturo J. Vegas ${ }^{1 *}$

${ }^{1}$ Department of Chemistry, Boston University, Boston, Massachusetts, United States

${ }^{2}$ Department of Biological Engineering, Massachusetts Institute of Technology, Cambridge, MA, United States

${ }^{3}$ Koch Institute for Integrative Cancer Research, Massachusetts Institute of Technology, Cambridge, MA, United States

${ }^{4}$ Broad Institute of MIT and Harvard, Cambridge, MA, United States

\section{Supplementary Information}




\section{Supplemental Tables and Figures}

Supplemental Table 1. List of proteins used to generate SMM binding signatures in Figure S1

\begin{tabular}{|l|l|}
\hline Protein Name & Protein Class \\
\hline Androgen Receptor Variant 7 (ARv7) & Transcription regulator \\
\hline BCL11AXL & Transcription regulator \\
\hline Bromodomain Containing Protein 4 (BRD4) & Transcription regulator \\
\hline Carbonic Anhydrase II (CAII) & Enzyme \\
\hline EWS/FLI & Transcription regulator \\
\hline Forkhead Box A1 (FOXA1) & Transcription regulator \\
\hline Forkhead Box P3 (FOXP3) & Transcription regulator \\
\hline High Mobility Group Nucleosome Binding Domain 1 (HMGN1) & Transcription regulator \\
\hline Heat Shock Factor 1 (HSF1_HA) & Transcription regulator \\
\hline Heat Shock Factor 1 (HSF1_His) & Transcription regulator \\
\hline Interleukin 4 (IL-4) & Cytokine \\
\hline B-ketoacyl ACP synthase I (KasA-His) & Enzyme \\
\hline MYB fragment & Transcription regulator \\
\hline MYB_MYBNFIB IVT & Transcription regulator \\
\hline Prion Protein (PrP) & Surface receptor \\
\hline Rev1 & DNA Polymerase \\
\hline SOX2 & Transcription regulator \\
\hline YAPD1 & Transcription regulator \\
\hline Yes-Associated Protein (YAP-HA) & Transcription regulator \\
\hline B-Cell Lymphoma/Leukemia 11A (BCL11A) & Transcription regulator \\
\hline Succinyl-CoA ligase [GDP-forming] subunit beta (Suclg2) & Enzyme \\
\hline MYC-Associated Protein X (Max) & Transcription regulator \\
\hline Chromodomain Helicase DNA Binding Protein 4 (CHD4) & Enzyme \\
\hline Glutathione S-transferases (GST) & Enzyme \\
\hline 3C-Like Protease (3CLpro) & Protease \\
\hline mp97 & Transcription regulator \\
\hline Sialic Acid-Binding Immunoglobulin-Like Lectin F (Siglec-F) & Glycan receptor \\
\hline Immunoglobulin G (IgG) & Antibody \\
\hline Siglec & Glycan receptor \\
\hline & \\
\hline
\end{tabular}


Supplemental Table 2. Control data for HEK-Blue IL-4/IL-13 inhibition experiments

\begin{tabular}{|l|l|}
\hline Condition & Abs650 \\
\hline Cells Alone & $0.0961 \pm 0.0113$ \\
\hline Cells with Vehicle & $0.0923 \pm 0.0079$ \\
\hline Cells with IL-4 and Vehicle & $1.391 \pm 0.0650$ \\
\hline Cells with 100 $\mu \mathrm{M}$ Compound 1 & $0.0955 \pm 0.0072$ \\
\hline Cells with 100 $\mu \mathrm{M}$ Compound 25 & $0.0648 \pm 0.0012$ \\
\hline Cells with 100 $\mu \mathrm{M}$ Compound 52 & $0.0588 \pm 0.0175$ \\
\hline
\end{tabular}

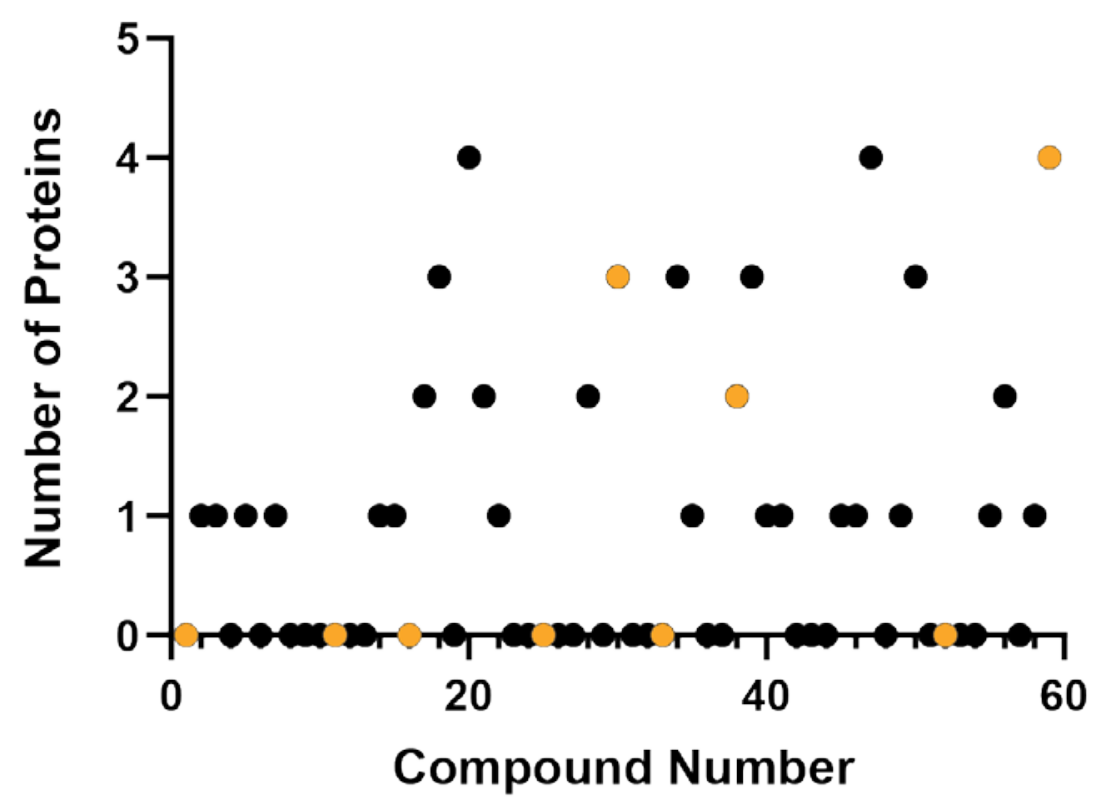

Supplemental Figure 1. SMM binding signatures for compounds 1-59 derived from SMM assays with >28 unrelated proteins. The number of proteins that these compounds were measured as binding positives is used as an indication of binding selectivity. The 10 compounds advanced into functional cell-based assays (Figure 2) are highlighted in orange. 


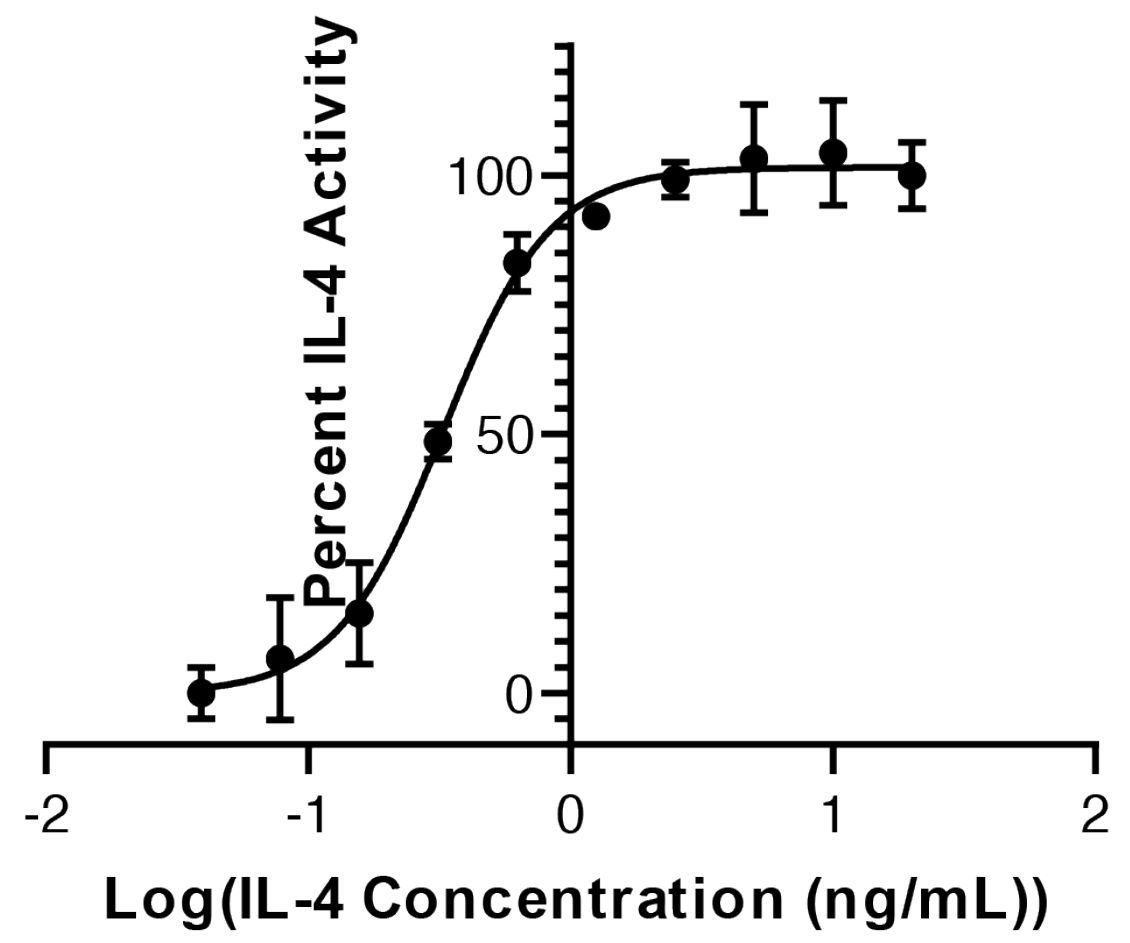

Supplemental Figure 2. Activity of IL-4 with $0.2 \%$ DMSO in HEK-Blue IL-4/IL-13 cells

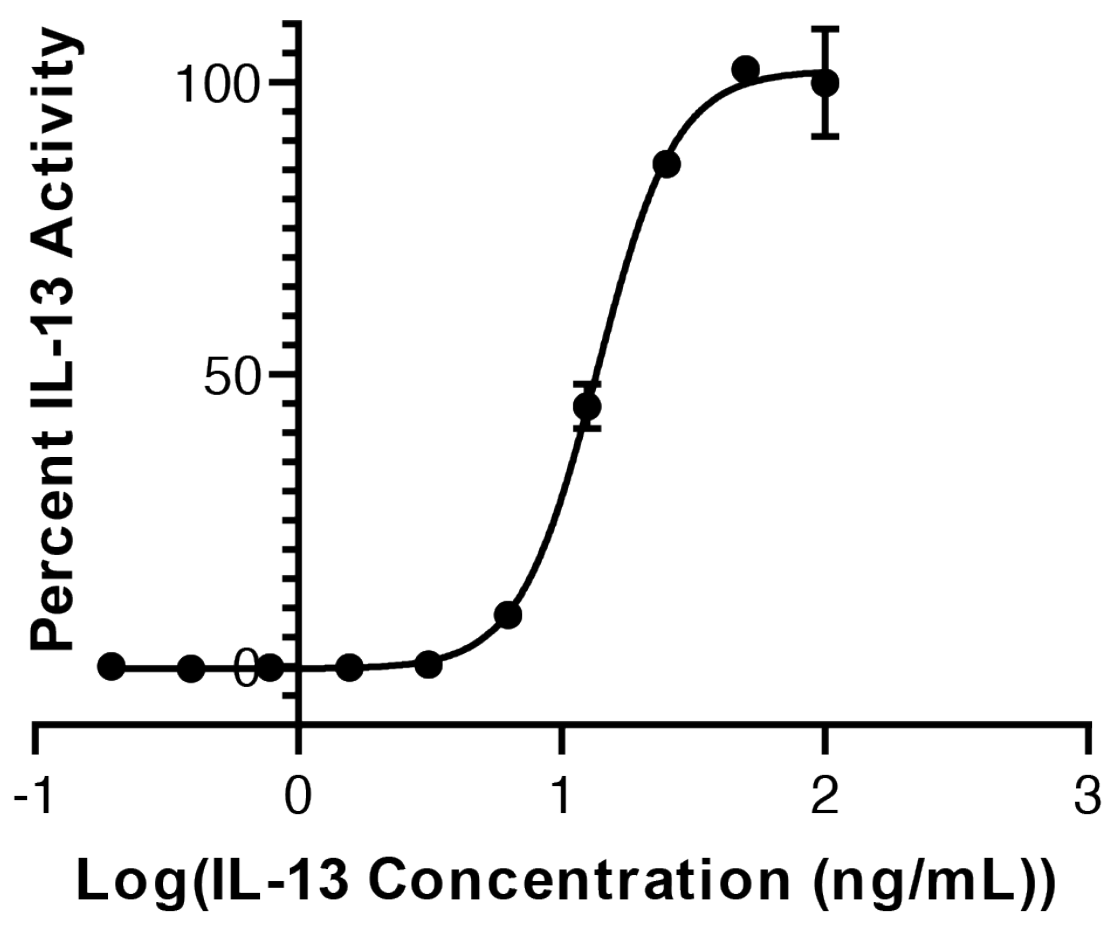

Supplemental Figure 3. Activity of IL-13 with $0.2 \%$ DMSO in HEK-Blue IL-4/IL-13 cells 


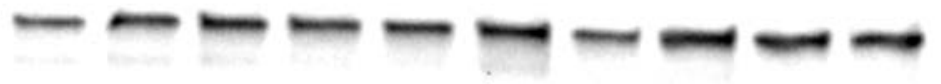

Supplemental Figure 4. Full STAT-6 Western Blot

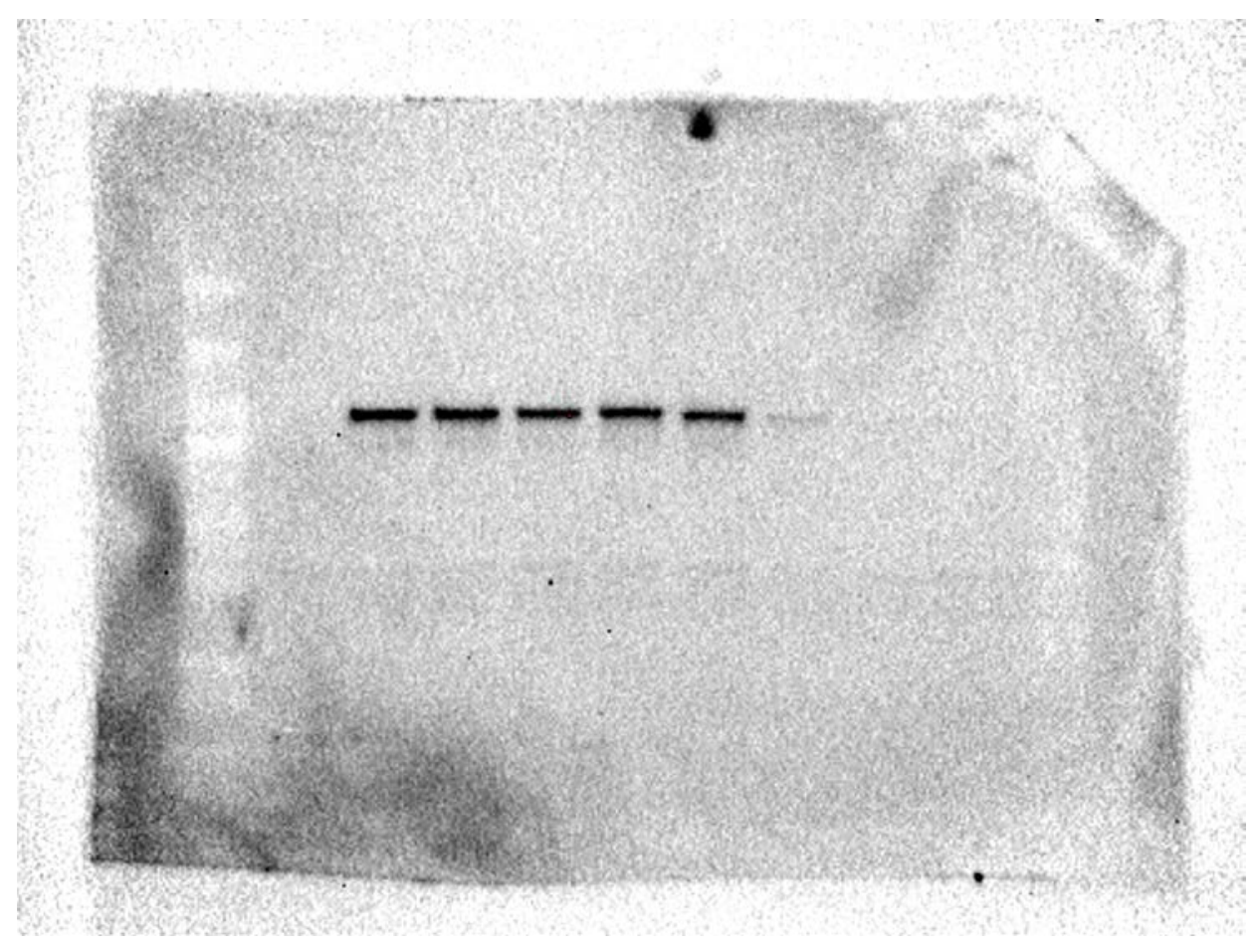

Supplemental Figure 5. Full p-STAT-6 Western Blot 


\section{Materials and Methods}

\section{Reagents}

His-tagged mammalian-expressed IL-4 for SMM and DSF was purchased from Abcam (ab185869). Tag-free HEK-293 expressed IL-4 for HEK-Blue IL-4/IL-13 and THP-1 inhibition assays was purchased from Acros Biosystems (IL4-H4218). Avitag IL-4 for surface plasmon resonance was purchased from Acros Biosystems (IL4-H82E0). Blasticidin, Zeocin, Normocin, Quanti-Blue was purchased from Invivogen. Commercial small molecules used in SMM were purchased from Chembridge. STAT-6 and p-STAT-6 antibodies were purchased from Cell Signaling Technology. All other chemicals and reagents were purchased from Fisher Scientific.

\section{SMM Screening of Recombinant IL-4}

Each SMM slide contained approximately 5000 printed features in duplicate and was prepared as described previously. ${ }^{1}$ In total 50,000 compounds were screened. The collection contained computationally selected commercially available compounds as well as products of diversity-oriented synthesis and known bioactive compounds. Each sample was screened against two replicate SMMs. Each slide was incubated with $3 \mathrm{~mL}$ of a solution of $0.1 \mu \mathrm{g} / \mathrm{ml}$ recombinant IL4-His protein in PBS-T buffer for 0.5 hour at room temperature. The slides were then incubated with a $3 \mathrm{ml}$ solution of anti-His mouse monoclonal antibody conjugated to AlexaFluor 647 (Qiagen) at a concentration of 1:1000. Each incubation step was followed by three washes in PBS-T. Finally the slides were briefly rinsed in distilled water and spin-dried. The slides were immediately scanned using a GenePix 4000B fluorescence scanner (Molecular Devices). The image was analyzed using GenePix Pro software (Axon Instruments) and data analyzed as described below. 


\section{SMM Statistical Analysis.}

Raw data was analyzed based on the signal-to-noise ratio and reproducibility. For each feature, the signal to noise ratio (SNR) was defined as the median fluorescence intensity of the feature divided by the median fluorescence intensity of the surrounding slide area, defined as a radius 3 times the radius of the spot, excluding pixels within a certain overlap threshold of neighboring features. Then, for each feature, a robust z-score $\left(z_{i}\right)$, which is less influenced by outliers compared with the mean-based $z$ statistic, was calculated for each feature $(\boldsymbol{i})$ by the following equation:

$$
\mathrm{z}_{\mathrm{i}}=\frac{\mathrm{SNR}_{\mathrm{i}}-M d n(\mathrm{SNR})}{M A D(\mathrm{SNR}) * 1.486}
$$

Where $\mathrm{SNR}_{\mathrm{i}}$ is the SNR value for a given feature, $M d n(\mathrm{SNR})$ is the median of the SNR values for all features in the subarray, and the $M A D(S N R)$ is the maximum absolute deviation of the SNR values for all features in the subarray. Assay positives with an average robust $Z$ score greater than 1.645 (alpha $=0.05)$ on duplicate slides were compared to both a counter screen for the detection antibody and a database representing $>25$ unique targets screened by SMM against the same chemical library to filter nonspecific binders.

\section{DSF}

Top hits from SMM were made into $1 \mathrm{mM}$ stock solutions in pure DMSO and dispersed in 384-well plates. $1 \mu \mathrm{g}$ of protein in PBS was added to each well, then 8X Spiro-Orange was added, followed by PBS, DMSO, or small molecules. Small molecules were screened 
at either $20 \mu \mathrm{M}$ or $2 \mu \mathrm{M}$ in $0.2 \%$ DMSO. DSF was performed on a BioRad CFX384. Change in melting temperature was calculated by comparing the nadir of the melting curve of protein with vehicle to the nadir of the melting curve of protein with small molecule. Plots were made in Graphad Prism 8.

\section{HEK-Blue IL-4/IL-13 Inhibition Assays}

HEK-Blue IL-4/IL-13 cells were purchased from Invivogen and maintained in DMEM complete with $10 \%$ fetal bovine serum, $10 \mu \mathrm{g} / \mathrm{ml}$ of blasticidin and $100 \mu \mathrm{g} / \mathrm{ml}$ of Zeocin, $100 \mathrm{ug} / \mathrm{mL}$ Normocin, and 100U/mL-100 $\mu \mathrm{g} / \mathrm{mL}$ Pen-Strep. Cells used for assays were between passages 12 and 18. Prior to screening small molecules, the SEAP production induced by IL-4 alone was tested in the cells (Figure S2) and determined to be $0.3 \mathrm{ng} / \mathrm{mL}$. The EC25 for the IL-4 induced SEAP production ( $0.1 \mathrm{ng} / \mathrm{mL}$ final well concentration) was chosen for running small molecule inhibition assays.

For screening small molecules, $25 \mu \mathrm{L}$ of each molecule (Figure 2) in DMEM with $4 \%$ DMSO was added to $25 \mu \mathrm{L}$ of $1 \mathrm{ng} / \mathrm{mL}$ IL-4 in DMEM and incubated for 2 hours at $37^{\circ} \mathrm{C}$. HEK-Blue IL-4/IL-13 cells were trypsinized, spun down, and resuspended in DMEM with $10 \%$ heat-inactivated FBS, and $100 \mathrm{U} / \mathrm{mL}-100 \mu \mathrm{g} / \mathrm{mL}$ Pen-Strep at a concentration of 3.125E $5 / \mathrm{mL} .160 \mu \mathrm{L}$ of the cell suspension was added to each well of a 96 well plate, avoiding the edges of the plate and equilibrated in an incubator for 1 hour. After 2 hours, $40 \mu \mathrm{L}$ of the small molecule/IL-4 solution was added to each well. Each measurement was performed in triplicate. Cells were incubated for 20 hours at $37^{\circ} \mathrm{C} / 5 \% \mathrm{CO}_{2}$. QuantiBlue was prepared according to manufacture and $160 \mu \mathrm{L}$ added to each well of a 96 well plate, avoiding edges. $40 \mu \mathrm{L}$ of cell supernatant was added to each well and then incubated for 3 hours at $37^{\circ} \mathrm{C}$. The optical density was then read at $650 \mathrm{~nm}$ with a 
Molecular Devices SpectraMax M5 Microplate Reader. Data was plotted and analyzed in GraphPad Prism 8.

Inhibition assays with IL-13 were performed similarly. First, the activity of IL-13 in HEK Blue IL-4/IL-13 cells was tested at concentrations from $0.2-100 \mu \mathrm{g} / \mathrm{mL}$ (final well concentrations) with $0.2 \%$ DMSO (Figure S3). $8 \mathrm{ng} / \mathrm{mL}$ was then incubated with increasing concentrations of D9 in $2 \%$ DMSO for 2 hours at $37^{\circ} \mathrm{C}$ before being added to cells. The supernatant was assayed for SEAP with Quanti-Blue and then absorbance taken at $650 \mathrm{~nm}$. Data was again plotted and analyzed in GraphPad Prism 8.

\section{THP-1 STAT-6/pSTAT-6 assay}

THP-1 cells were received as a gift from the laboratory of Dr. Mark Grinstaff and maintained in RMPI-1640 with $10 \%$ heat-inactivated fetal bovine serum and $50 \mu \mathrm{M}$ betamercaptoethanol. Cells used for assays were between passages 6 and $10.10 \mathrm{ng} / \mathrm{mL} \mathrm{IL}$ 4 was preincubated with experimental sample (vehicle or small molecules) for 2 hours at $37^{\circ} \mathrm{C}$. $1 \mathrm{~mL}$ of each solution was then added to a pellet of 2E 6 THP-1 cells and pipetted up and down. THP-1 cells were incubated for 30 minutes in incubator at $37^{\circ} \mathrm{C} 5 \% \mathrm{CO} 2$. Cells were then spun down, lysed with $200 \mu \mathrm{L}$ ice-cold RIPA buffer complete with phosphatase and protease inhibitors. Cell lysate was incubated on ice for 30 minutes and then stored at $-20^{\circ} \mathrm{C}$ until western blot analysis. Undiluted cell-lysate was run SDS-PAGE followed by transfer to membrane using Biorad equipment. Membranes were incubated with 1:1000 primary antibody overnight at $4^{\circ} \mathrm{C}$. Membranes then incubated with $1: 50,000$ dilution secondary for 1 hour at RT. Membranes were then exposed to Femto ECL for 5 minutes and imaged using a Bio-Rad Gel Doc. The brightness/contrast was adjusted in FIJI (Figures S4 and S5) and the area under the curve of each lane was quantified. The 
ratio of phosphorylated STAT6 to STAT6 was calculated and normalized to the amount of total STAT6. The data was plotted and analyzed using Graphpad Prism 8. Immunofluorescence for pSTAT-6

THP-1 cells were plated at a density of $1.5 \mathrm{E} 6 / \mathrm{mL}$ into a MatTak dish coated with Cell Tak adhesive. Cells were allowed to attach for 1 hour. IL-4 and small molecule or vehicle (DMEM with 2\% DMSO) was pre-incubated for 2 hour at $37^{\circ} \mathrm{C}$. Media was aspirated and IL-4 or IL-4/small molecule were added to cells and incubated for 30 minutes at $37^{\circ} \mathrm{C} 5 \%$ CO2. Cells were rinsed twice with PBS and fixed with $2 \%$ formaldehyde for 30 minutes at room temperature. Cells were rinsed twice and then permeabilized with $0.1 \%$ Triton- $X$ for 10 minutes. Cells were blocked for 1 hour in superblock with $0.05 \%$ Tween-20, rinsed twice, and then incubated with anti-pSTAT6 1:100 diluted in SuperBlock 0.05\% Tween20 overnight at $4^{\circ} \mathrm{C}$. Cells were rinsed three times with PBS $0.05 \%$ Tween-20 and then incubated with Goat anti-rabbit IgG $(\mathrm{H}+\mathrm{L})$ Secondary Antibody - Alexa Fluor 647 mixed with a 1:250 dilution of Hoechst 33342. Cells were rinsed twice and left in $1 \mathrm{~mL}$ of PBS. Confocal imaging was done using an Olympus FV10i and images were processed using FIJI.

\section{Surface plasmon resonance (SPR)}

Surface plasmon resonance was performed on a Biacore T200. SA sensor chips were purchased from GE Healthcare. The running buffer was 1X PBS pH 7.40 with $2 \%$ DMSO and $0.1 \%$ Tween-20 and the flow rate was kept constant at $50 \mu \mathrm{L} / \mathrm{min}$ unless otherwise noted. The SA chip was activated with three 1 minute injections of $1 \mathrm{M} \mathrm{NaCl}$ in $50 \mathrm{mM}$ $\mathrm{NaOH}$ followed by a wash of $50 \%$ isopropanol in $1 \mathrm{M} \mathrm{NaCl}$ and $50 \mathrm{mM} \mathrm{NaOH} .50 \mu \mathrm{L}$ of a $5 \mathrm{mg} / \mathrm{mL}$ solution of Avitag-IL-4 in DMSO free buffer was injected to immobilize $3000 \mathrm{RU}$ 
on the chip surface using the software wizard. Two manual $50 \mu \mathrm{L}$ injections of $50 \mathrm{mM}$ biocytin dissolved in running buffer were then performed to block all biotin sites on the sensor chip. The system was then primed with running buffer for 10 minutes. Compound 8 was diluted in running buffer and injected for 300 second cycles in triplicate for each concentration, starting from low concentration. The dissociation phase was 600 seconds long. Sensogram figures were created by exporting the raw data and plotting in Graphpad Prism 8.

\section{References}

(1) Bradner, J. E.; McPherson, O. M.; Koehler, A. N. A Method for the Covalent Capture and Screening of Diverse Small Molecules in a Microarray Format. Nat. Protoc. 2006, 1 (5), 2344-2352. https://doi.org/10.1038/nprot.2006.282. 